\title{
TERATOMA PLACENTARIO: REPORTE DE UN CASO Y REVISIÓN DE LA LITERATURA
}

\author{
Placental teratoma: Case report and review \\ of the literature \\ Diego Armando García-Riaño, MD ${ }^{1}$; Rafael Leonardo Aragón-Mendoza, MD²; \\ Claudia Patricia Méndez-Sarmiento, $M^{3}$
}

Recibido: noviembre 5/13 - Aceptado: septiembre 12/14

\section{RESUMEN}

Objetivo: el teratoma placentario es un tumor benigno poco frecuente, solo han sido reportados algunos casos a nivel mundial, ninguno de estos en Colombia. Se presenta un caso para revisar la literatura publicada respecto a su diagnóstico prenatal, histopatológico y pronóstico en el embarazo. Materiales y métodos: se presenta el caso de una paciente de 19 años, con gestación de 32 semanas, quien consultó a la Unidad de Obstetricia del Hospital Universitario Clínica San Rafael, hospital general privado de IV nivel de complejidad en atención, ubicado en Bogotá (Colombia). La paciente tenía como hallazgo ultrasonográfico prenatal una imagen de ecogenicidad heterogénea en cavidad amniótica. Se encontró una masa ovoide, de aspecto sólido, de 5 centímetros, localizada en la cara fetal de la placenta. El estudio histopatológico mostró hallazgos correspondientes a teratoma placentario. Se realiza una revisión de la literatura publicada en las bases de datos: PubMed, Lilacs, SciELO, Ovid y ScienceDirect.

1 Médico especialista en Ginecología y Obstetricia, Unidad de Ginecología y Obstetricia, Hospital Universitario Clínica San Rafael, Bogotá (Colombia).dgarciamd@gmail.com.

2 Médico especialista en Ginecología y Obstetricia, Universidad Militar Nueva Granada, Hospital Universitario Clínica San Rafael, Bogotá (Colombia).

3 Médico especialista en Patología, Hospital Universitario Mayor Mederi, Bogotá (Colombia).
Resultados: se encontraron 10.523 títulos, de los cuales se seleccionaron 23 relacionados con teratoma placentario. Todos eran reportes de caso. $\mathrm{Su}$ diagnóstico se basa en la ecografía prenatal y en el estudio histopatológico, que muestra tejidos maduros de las tres capas germinativas sin organización definida. Tiene un buen pronóstico obstétrico y no parece incrementar el riesgo de malformaciones fetales.

Conclusión: el teratoma placentario es un tumor de aparición poco frecuente, que generalmente tiene un comportamiento benigno.

Palabras clave: placenta, teratoma, neoplasia.

\section{ABSTRACT}

Objective: Placental teratoma is an infrequent benign tumour and there are only a few reports in the world, none of them in Colombia. A case is presented with a review of the literature regarding prenatal and histopathology diagnosis, and prognosis of placental teratoma in pregnancy. Materials and methods: We present the case of a 19 year-old patient in week 32 of gestation who came to the Obstetric Unit at the Hospital Universitario San Rafael, a private Level IV general hospital in Bogotá, Colombia. The patient had a prenatal ultrasound finding of an image of heterogeneous echogenicity in the amniotic cavity. An oval-shaped solid-appearing 5-cm mass 
was found on the fetal side of the placenta. The histopathology showed findings consistent with placental teratoma. A review of the literature was conducted in the PubMed, Lilacs, SciELO, Ovid, and ScienceDirect databases.

Results: Of 10,523 titles found, 23 related to placental teratoma were selected. They were all case reports. The diagnosis of placental teratoma is based on prenatal diagnosis and histopathology; the latter shows mature tissues of the three germinal layers without defined organization. It has a good obstetrical prognosis and does not appear to increase the risk of foetal malformations.

Conclusion: Placental teratoma is a tumor of infrequent ocurrence, usually of benign nature.

Key words: Placenta, teratoma, neoplasia.

\section{INTRODUCCIÓN}

Los tumores de la placenta son poco frecuentes. Se dividen en trofoblásticos y no trofoblásticos, estos últimos a su vez en primarios y secundarios. El tumor placentario no trofoblástico y primario más común es el corangioma, que se presenta en un $1 \%$ de todos los embarazos $(1,2)$. El segundo tumor placentario no trofoblástico y primario es el teratoma, descrito por primera vez en 1925 por P. Morville (3). Desde entonces, en la literatura solo han sido reportados pocos casos a nivel mundial (4).

El origen del teratoma placentario no es claro (5), pero existen dos hipótesis: la primera, descrita por Fox y Butler (6), la cual sugiere que en las primeras etapas embrionarias se presenta una migración de las células pluripotenciales desde la pared dorsal del saco vitelino hacia el intestino primitivo y se depositan en el tejido conectivo del cordón umbilical. Eventualmente pasan al tejido conectivo entre el amnios y la superficie fetal de la placenta (2, 5, 7, 8). La segunda, considera el teratoma placentario como una variante degenerativa del gemelo acárdico (9-12).

Este tumor contiene tejidos no organizados de las tres capas germinativas. Se ubica principalmente entre el amnios y el corion, pero también puede estar en la superficie placentaria fetal, dentro de las membranas fetales y en el cordón umbilical (13-15).

El teratoma placentario es una patología con pocos casos reportados a nivel mundial, por lo que decidimos presentar este caso con el objetivo de realizar una revisión y aportar al tema en aspectos como diagnóstico prenatal, histopatológico y pronóstico en el embarazo.

\section{REPORTE DE CASO}

Paciente de 19 años, G2A1P0 con gestación de 32 semanas, que consultó al servicio de urgencias del Hospital Universitario Clínica San Rafael, centro de IV nivel de complejidad en atención, al que asisten pacientes afiliados al régimen contributivo del sistema de seguridad social en Colombia. Tenía diagnóstico previo de preeclampsia en semana 30 dado por elevación de tensión arterial y proteinuria en 24 horas de $310 \mathrm{mg}$. Se encontraba en seguimiento obstétrico semanal. En el momento de la consulta se diagnosticó una preeclampsia severa y se decidió hospitalización. La paciente tenía estudios ecográficos prenatales que mostraban una adecuada curva de crecimiento fetal. El último, realizado en la semana 31, evidenciaba una imagen redondeada en cavidad amniótica, heterogénea, con un componente quístico, además de algunos tabiques y calcificaciones. No guardaba relación con la placenta ni con estructuras fetales. Las medidas reportadas fueron 55 x 50 milímetros (figura 1). No se realizó estudio doppler complementario en el momento. Una vez hospitalizada, se inició el manejo con antihipertensivo, sulfato de magnesio y esquema de maduración pulmonar fetal. Durante la evolución la paciente presentó disnea, taquipnea y requerimiento de oxígeno suplementario. El examen físico y radiológico confirmó el diagnóstico de edema pulmonar. Posteriormente se realizó manejo con diurético y, una vez completado el esquema de maduración pulmonar fetal, la paciente fue llevada a cesárea. Se obtuvo un recién nacido femenino, sin malformaciones aparentes. Su peso fue $1785 \mathrm{~g}$, midió 44 centímetros, los puntajes de Apgar fue- 


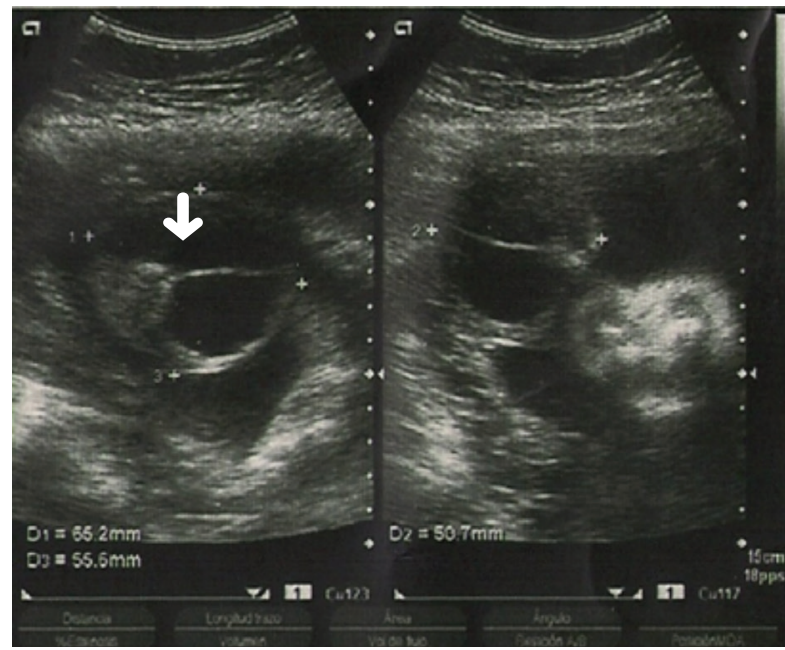

Figura 1. Imagen redondeada en cavidad amniótica, con componente quístico, tabiques y calcificaciones (flecha)

ron: 7 al minuto y 5 a los 5 minutos, y el puntaje de Ballard fue compatible con una edad gestacional de 32 semanas. Como hallazgo quirúrgico adicional se encontró una placenta fúndica, sin alteraciones en su inserción, y adyacente a esta había una masa sólida, ovoide, color amarillo pardo, con superficie regular y lisa, de $5 \mathrm{~cm}$ aproximadamente, y estaba apenas adherida a la placenta. Inicialmente fue interpretada como un lóbulo placentario accesorio y se envió a estudio histopatológico. La paciente presentó una adecuada evolución posoperatoria, con alta médica al controlar sus cifras tensionales con manejo antihipertensivo. La recién nacida requirió atención en unidad de cuidado intensivo neonatal, por síndrome de dificultad respiratoria con evolución satisfactoria, y alta médica en plan canguro.

El estudio patológico reportó una placenta de tamaño normal para la edad gestacional, con peso ligeramente elevado y cordón umbilical de aspecto normal. Se encontró una formación ovoide de $6 \times 5 \mathrm{~cm}$, localizada en la cara fetal y adherida a membranas fetales, sin pedículo ni cordón umbilical (figura 2). La superficie externa corresponde a piel parcialmente cubierta de vello. Al corte se evidenció un área sólida de 3 x 3 centímetros, de color pardo claro, con áreas amarillas granulares y una zona quística de 3 x 2 centímetros, con contenido sanguinolento. La microscopia mostró una placenta madura del tercer trimestre, con cambios compatibles con enfermedad hipertensiva del embarazo dados por infartos placentarios agudos y antiguos. El estudio histológico de la lesión reveló la presencia de piel y anexos, grasa parda, epitelio escamoso inmaduro, tejido compatible con plexos coroides, tejido conectivo, músculo liso y epitelio respiratorio (figura 3). Teniendo en cuenta estos hallazgos, y ante la ausencia de esqueleto axial y extremos caudal o cefálico, se realizó el diagnóstico de teratoma placentario.

Aspectos éticos. Se solicitó autorización a la paciente y al Hospital Universitario Clínica San Rafael para publicar las fotos. Se garantizó la confidencialidad para proteger los derechos de la paciente.

\section{MATERIALES Y MÉTODOS}

Se realizó una búsqueda bibliográfica en las bases de datos electrónicas de: Medline vía PubMed, Lilacs, SciELO, Ovid y ScienceDirect con los términos "placenta", "teratoma” y "neoplasia”. Se seleccionaron artículos publicados en inglés y español, desde 1990 a julio 2014, que describieran diagnóstico ecográfico, histopatológico y pronóstico obstétrico del teratoma placentario.

\section{RESULTADOS}

Se encontraron 10.523 títulos, de los cuales se seleccionaron 23 relacionados con teratoma placentario. Todos fueron reportes de caso.

Diagnóstico por ultrasonido. El diagnóstico prenatal se realiza a través de ultrasonido. Se describen como lesiones circunscritas a nivel de la placenta o adyacentes a esta, de contenido sólido y quístico con diferentes ecogenicidades, sin evidencia de cordón umbilical. Se pueden presentar solo quísticas en un 10 a 20\%, y hasta en un 40\% de los casos se evidencian calcificaciones. Este último hallazgo es importante, ya que no se ha reportado en otros tumores placentarios más frecuentes como los de tipo trofoblástico y el corangioma (4). Esto se podría explicar por el origen de células pluripotenciales 


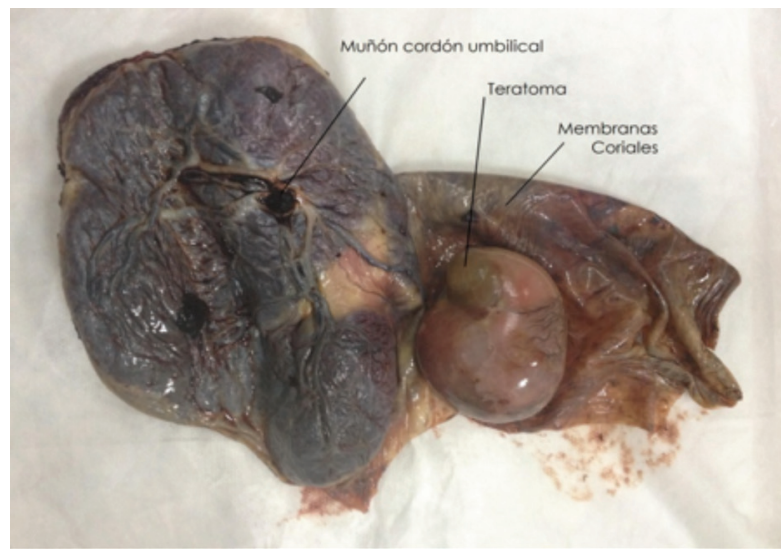

Figura 2. Hallazgos macroscópicos
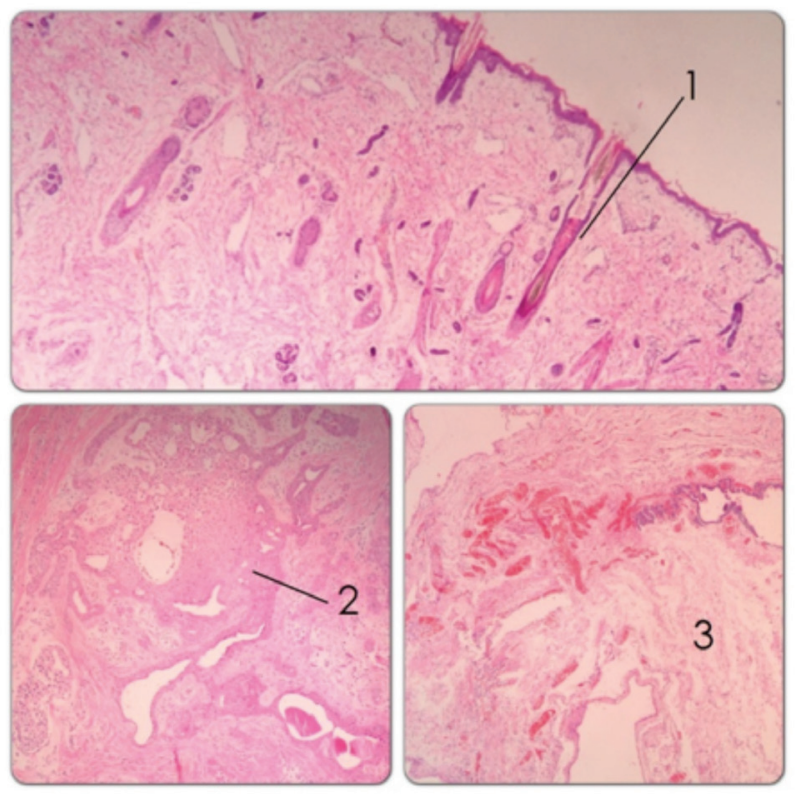

Figura 3. Hallazgos microscópicos: 1) anexos cutáneos, 2) tejido epitelial, 3) tejidos conectivos y estructuras compatibles con plejos coroides

del teratoma placentario que derivan a un tejido maduro calcificado.

Diagnóstico histopatológico. Estos tumores miden entre 2 y 11 centímetros. La mayor parte de los casos reportados fueron sólidos con solo una excepción, en la que el teratoma fue descrito como quístico exclusivamente (9). En casi todos los casos se localizan entre el amnios y el corion, aunque también se pueden ubicar en la superficie placentaria fetal, dentro de las membranas fetales o incluso dentro del tejido placentario (16). Carecen de cordón umbilical, pero pueden tener un pedículo vascular no recubierto de membrana amniótica ni gelatina de Wharton.

En el microscopio se evidencian tejidos maduros derivados de las tres capas germinativas sin organización definida $(4,17)$, aunque recientemente se reportó un caso en donde se evidencia un teratoma placentario inmaduro con hallazgos de necrosis y elementos neuroepiteliales mezclados con tejido maduro e inmaduro de origen ectodérmico y mesodérmico (5).

El principal diagnóstico diferencial del teratoma placentario es el gemelo acárdico. Este se caracteriza por iniciar como una gestación múltiple monocorial identificada como hallazgo sonográfico. En el estudio histopatológico se encuentra cordón umbilical que normalmente es bivascular, además de la presencia de un desarrollo axial, es decir, la identificación de un polo craneal o caudal, columna vertebral, costillas, pelvis, base de cráneo, formación de órganos o extremidades reconocibles $(4,7,9,17-23)$. Puede ser útil una radiografía de la lesión para esclarecer estos hallazgos.

Pronóstico. El reconocimiento prenatal del teratoma placentario es útil, ya que la mayoría tiene un comportamiento benigno y no parece incrementar el riesgo de malformaciones congénitas o comprometer el curso normal del embarazo (18, 24-26). Sin embargo, se han reportado casos aislados de malformaciones fetales asociadas tales como mielomeningocele $(5,25)$. Esto puede ser interpretado como hallazgo no relacionado con la fisiopatología del teratoma placentario.

En 1991, Akimov describió un único caso aislado de un gran teratoma placentario que resultó en asfixia fetal, posiblemente debido a la compresión mecánica del cordón umbilical (27). En consecuencia, en masas mayores a 10 centímetros se recomienda hacer un seguimiento estricto prenatal e intraparto del bienestar fetal, por la posibilidad de hipoxia fetal (10). 


\section{CONCLUSIÓN}

El teratoma placentario es un tumor de aparición esporádica. El diagnóstico prenatal se basa en hallazgos ecográficos de lesiones placentarias o adyacentes a esta, con diferente ecogenicidad y calcificaciones en el $40 \%$ de los casos. En el estudio histopatológico se encuentran tejidos maduros de las tres capas germinativas sin organización definida. Su principal diagnóstico diferencial es el gemelo acárdico, para lo cual se debe tener en cuenta la historia obstétrica, la ausencia de cordón umbilical y la falta de organización axial como características distintivas. La mayoría tiene un comportamiento benigno, que no parece incrementar el riesgo de malformaciones fetales ni desenlaces perinatales adversos. La información acerca de esta patología es muy limitada debido a los escasos reportes en la literatura mundial.

\section{REFERENCIAS}

1. Sarmiento A, Quijano FE, Puccini G, Rodríguez N, Gómez PA. Corangioma placentario. Reporte de caso y revisión de la literatura. Rev Colomb Obstet Ginecol. 2008;59:62-7.

2. Gruca K, Ropacka M, Breborowicz GH. Nontrophoblastic placental tumors. Archives of Perinatal Medicine. 2011;17:113-7.

3. Morville P. Une teratoma placentaire. Obstet Gynecol. 1925;11:29-32.

4. Kudva R, Monappa V, Rai L. Placental teratoma: a diagnostic dilemma with fetus acardius amorphous. Indian Journal of pathology and microbiology. 2010; 53:378-9.

5. Shahzadi S. Immature placental teratoma. Journal of postgraduate medical institute. 2014;28:324-7.

6. Fox H, Butler-Manuel R. A Teratoma of the Placenta. The Journal of Pathology and Bacteriology. 1964; 88:137-140.

7. Prashanth A, Lavanya R, Girisha KM, Mundkur A. Placental Teratoma presenting as a lobulated mass behind the neck of fetus: a case report. Case Rep Obstet Gynecol. 2012;2012:857230.

8. Nagehan O, Cem C, Sevinc K, Aylin E. Mature placental teratoma: case report. Open Journal of Pathology. 2013;3:131-2.
9. Chandy R, Korula A, Seshadri L. Teratoma of the placenta. Australian and New Zealand Journal of Obstetrics and Gynaecology. 2002;42:556-7.

10. Tan G, Shiran M, Aireen I, Swaminathan M, Hayati A, Zaleha A. Large teratoma of the Placenta. The Internet Journal of Gynecology and Obstetrics. 2008;11:12.

11. Elagoz S, Akera H, Cetin A. Placental teratoma: a case report. European Journal of Obstetrics \& Gynecology and Reproductive Biology. 1998;80:263-5.

12. Jamal AA. Placental teratoma: a case report and review of the literature. Annals of Saudi Medicine. 1999;19:359-61.

13. Hargitai B, Csabai L, Bán Z, Hetényi I, SzucsI, Varga $\mathrm{S}$, et al. Rare case of exomphalos complicated with umbilical cord teratoma in a fetus with trisomy 13 . Fetal Diagnosis and Therapy. 2005;20:528-33.

14. Satgé DC, Laumond MA, Desfarges F, Chenard MP. An umbilical cord teratoma in a 17 week old fetus. Prenatal Diagnosis. 2001;21:284-8.

15. Kreczy A, Alge A, Menardi G, Gassner, Gschwendtner A, Mikuz G. Teratoma of the umbilical cord. Case report with review of the literature. Arch Pathol Lab Med. 1994;118:934-7.

16. Unger JL. Placental Teratoma. American Journal of Clinical Pathology. 1989;92:371-3.

17. Gaffar BA, Hotait HY, Gamlouch SF, Swalaha NJ. Placental teratoma or fetus acardius amorphous? Hematology/Oncology and Stem Cell Therapy. 2008;1:57-61.

18. Ahmed N, Kale V, Thakkar H, Hanchate V, Dhargalkar P. Sonographic diagnosis of placental teratoma. J Clin Ultrasound. 2004;32:98-101.

19. Meinhard K, Dimitrov S, Nicolov A, Dimitrova V, Vassilev N. Placental teratoma - a case report. Pathol Res Pract. 1999;195:649-51.

20. Tzelepi V, Zolota V, Mavromati E. Fetus amorphous acardious: report of a rare case and differential diagnosis from placental teratoma with review of the literature. Eur Rev Med Pharmacol Sci. 2007;11: 419-22.

21. Molina R, Ávila de la Paz C, Martínez E. Feto acárdico amorfo o teratoma placentario. Revista Obstetricia y Ginecología Venezuela. 1994;54:101-4.

22. Shimojo H, Itoh N, Shigematsu H, Yamazaki T. Mature teratoma of the placenta. Pathol Int. 1996;46:372-5. 
23. Block D, Cruikshank S, Kelly K, Stanley M. Placental teratoma. Int J Gynaecol Obstet. 1991;34:377-80.

24. Dangela AR, Atlasa RO, Matsuoa BK. Prenatal diagnosis of placental teratoma: a case report. European Journal of Obstetrics \& Gynecology and Reproductive Biology. 2009;146:232-6.

25. Wang L, Du X, Li M. Placental teratoma. A case report and review of the literature. Pathology Research and Practice. 1995;191:1267-70.
26. Anuradha K, Thomas S, Revadi PS, Muley PR. A case report of placental teratoma: an unusual primary non-trophoblastic tumor of placenta. Indian J Pathol Microbiol. 2005;48:223-4.

27. Akimov OV. Giant teratoma of the placenta (acardius amorphus). Arkhiv Patologii. 1991;53:59-60.

Conflicto de intereses: ninguno declarado. 